\title{
High performance Digital Optical Switch
}

\author{
G. Singh, ${ }^{* 1}$ S. Gupta, ${ }^{1}$ P. Jain, ${ }^{2}$ S. Bothra, ${ }^{1}$ V. Janyani, ${ }^{1}$ R.P. Yadav ${ }^{1}$ \\ ${ }^{I}$ Department of Electronics and Communication Engineering, Malaviya National Institute of Technology Jaipur \\ ${ }^{2}$ Graduated from Modi Institute of Technology Lakhshamangarh, Rajasthan
}

Received February 18, 2011; accepted March 11, 2011; published March 31, 2011

\begin{abstract}
In this work, we present a high performance Y-shaped digital optical switch (DOS) which complies with the DOS reported earlier using Titanium in-diffused or proton exchanged Lithium Niobate waveguiding structures. The optimization of switching characteristics is performed for switching voltage and device size. This structure has a total length of $5.5 \mathrm{~mm}$ and a width of $20.6 \mu \mathrm{m}$. For the first time, the driving voltage as low as $1 \mathrm{~V}$ has been achieved with acceptable crosstalk levels (in the range of $-22 \mathrm{~dB}$ to $-28 \mathrm{~dB}$ ) for communication applications. Using a beam propagation method (BPM), we demonstrate that the switch is polarization independent and suitable for low attenuation optical window $(1300 \mathrm{~nm}$ and $1550 \mathrm{~nm})$ operation as well.
\end{abstract}

In the modern age of communication, switches are one of the most important devices in optical networks. Among various semiconductor switches, a digital optical switch (DOS) based on adiabatic mode evolution has experienced maximum attention due to its unique characteristics of low polarization and low wavelength sensitivity. A conventional Y-shaped waveguiding structure had been preferred to realize a DOS with polymers [1], amorphous $\mathrm{Si}$ [2-3], Ti-indiffused $\mathrm{LiNbO}_{3}$ [4-7] because of its easy and compact design, though it usually suffers from a high driving voltage and crosstalk (CT) levels. The improvements in CT had been proposed by modifying this structure, such as using double-etch waveguides [8], asymmetric X-junction [9], cascaded DOS [1] and a two-stage dilated switch [10].

However, the crosstalk suppression using these approaches had been achieved at the expense of higher driving voltages, while device operation with low power requirements is one of the key issues in modern communication networks. Therefore emphasis shall be given on the development of a DOS with low CT levels and switch losses for low power (voltage) requirements. Proper electrode positioning shall lead to electro-optic switches with reduced losses and driving power requirements as well [7], [11]. In this work, we propose a Y-shaped digital optical switch based on conventional structure with the lowest switching voltage (1V) and CT as low as in the range of $-22 \mathrm{~dB}$ to $-28 \mathrm{~dB}$. The device design and analysis have been done with the Beamprop software and beam propagation method (BPM),

\footnotetext{
*E-mail: gschoudhary75@gmail.com
}

respectively. We have concentrated on a Ti-indiffused or proton exchanged $\mathrm{LiNbO}_{3}$ electro-optic DOS utilizing optimized and properly shaped (slanted) electrode regions in accordance to output waveguides to achieve better switching performance with lower voltage requirements than previously reported.

Figure 1 depicts the layout of the proposed DOS based on a conventional $\mathrm{Y}$-shaped waveguiding structure. In our case, the channel waveguide width $\left(\mathrm{C}_{\mathrm{w}}\right)$ is $4 \mu \mathrm{m}$ and the entire structure has a length of $5.5 \mathrm{~mm}$ with a total device width of $20.6 \mu \mathrm{m}$. The length of the output arms (slanted waveguides, $\mathrm{L}_{\text {arm }}$ ) is $3.5 \mathrm{~mm}$, with a separation of $10 \mu \mathrm{m}$ between the end facets of its output ports. The optimum length of electrodes $\left(\mathrm{L}_{\mathrm{e}}\right)$ is found to be $3 / 4^{\text {th }}$ of the switch arms i.e. $2.62 \mathrm{~mm}$. Here we considered slanted shaped electrode regions in accordance with outer arms rather than choosing linear electrodes as previously used [11].

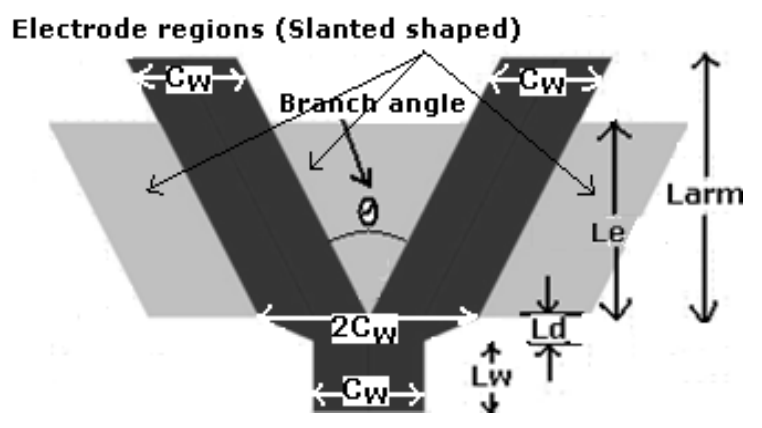

Fig. 1. Proposed 1x2 DOS layout.

Extensive iterations were performed to found the best value for a branch angle, with which the device operates as an efficient splitter under no bias conditions. Simple multilayer geometry has been considered for the electrodes and for each layer, the dielectric constant and electro-optic coefficient is defined for electrical field calculation. The value of a relative dielectric constant in $\mathrm{X}$ and $\mathrm{Y}$ directions is taken to be the index squared. The electro-optic coefficient value of $0.0008 \mu \mathrm{m} / \mathrm{V}$ is taken in the X-direction to enhance the device performance using a unidirectional concentrated electro-optic effect. 
Several material compositions and methods can be used to fabricate the waveguides for $\mathrm{Y}$ shaped DOS structures. Using electro-optic materials such as $\mathrm{LiNbO}_{3}$, one of the common methods is the Ti-indiffusion or proton exchanged process. By this process we have the bell shaped refractive index profile of a channel waveguide [11]. So here we are using the Gaussian profile of channel R.I. for a switch waveguide. We also check the results by assuming the step index profile of channel R.I. The index difference between cladding and channel is 0.01 . Index perturbation is computed from the following equation [12].

$$
\Delta \mathrm{n}=\left[\left(\frac{\left(\mathrm{N}_{0}\right) 3 \mathrm{R}_{x} \mathrm{E}_{x}}{2}+\frac{\left(\mathrm{N}_{0}\right) 3 \mathrm{R}_{y} \mathrm{E}_{y}}{2}\right)\right]
$$

Here $E_{x}$ and $E_{y}$ are the horizontal and vertical fields respectively from the electrodes. $\mathrm{R}_{\mathrm{x}}$ and $\mathrm{R}_{\mathrm{y}}$ are electrooptic coefficients measured in $\mu \mathrm{m} / \mathrm{V}$, while $\mathrm{N}_{0}$ is the optical index of the layer. Its value $\left(\mathrm{N}_{\mathrm{o}}\right)$ is equal to the background index which is 0.01 less than the refractive index of the material. For our calculation, the control parameter for electrodes is the applied voltage.

The device behaves as a half power splitter when no voltage is applied at the electrodes and behaves as a switch when voltage is applied. The device has been simulated with a 3D semi-vectorial approach using the beam propagation method for TE and TM polarized light propagation with both Step index and Gaussian index profiles. For optimizing the design of a DOS for size and crosstalk, first we set the applied voltage on electrodes as zero and by varying the branching angle and refractive index profile in the proper range, we obtain their optimum values as $0.1^{\circ}$ and 2.17 , respectively, for which the power splitter has the maximum efficiency for light propagation of both test wavelengths.

The device has been simulated for both TE and TM polarized mode at the test wavelengths of $1300 \mathrm{~nm}$ and $1550 \mathrm{~nm}$ and results are obtained accordingly. To optimize the switch performance, we have fixed the obtained values for the branching angle and R.I. of a channel waveguide and then the influence of electrode voltages in the range of $0 \mathrm{~V}$ to $5 \mathrm{~V}$ has been investigated to expect the best crosstalk levels (Fig. 2) and low switching losses such as insertion and excess losses (Fig. 3 and Fig. 4).

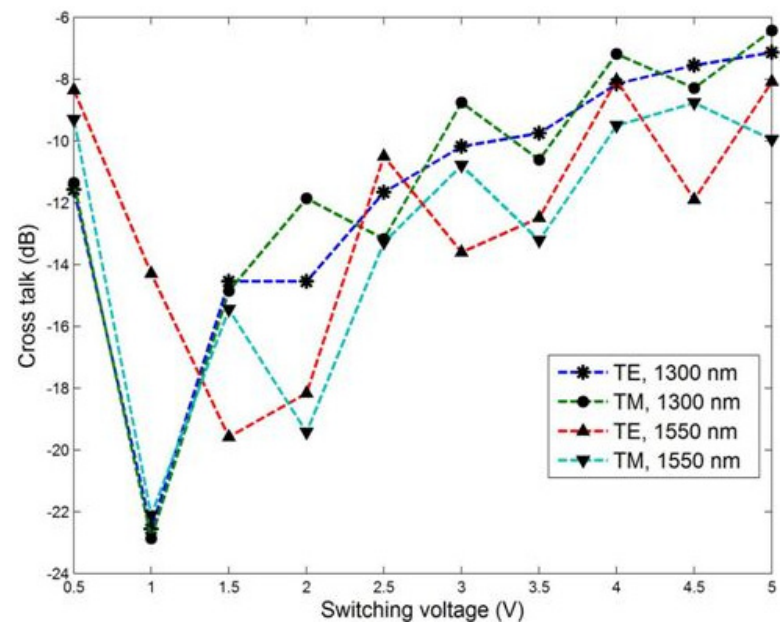

Fig. 2. Variation of crosstalk levels (C.T in dB) w.r.t applied voltages across electrode regions.

With these graphs, we can easily conclude that the best switching voltage for the given design is $1 \mathrm{~V}$ as we got the crosstalk of $-23 \mathrm{~dB}$ for TE polarization at $1.3 \mu \mathrm{m}$ and also for other polarization and wavelengths we got the crosstalk of around $-22 \mathrm{~dB}$. Also the switch losses such as I.L and E.L are in the acceptable range for $1 \mathrm{~V}$. In the next step, the effect of a branching angle and channel R.I. on switch CT levels were studied with the aid of $3 \mathrm{D}$ simulations, while the driving voltage was kept the same at $1 \mathrm{~V}$ for all cases. It has been observed that we can further reduce the crosstalk by increasing the channel index and branching angle (up to $0.13^{\circ}$ ). The crosstalk levels for the switch w.r.t to increased index profile and with a larger branch angle were calculated.

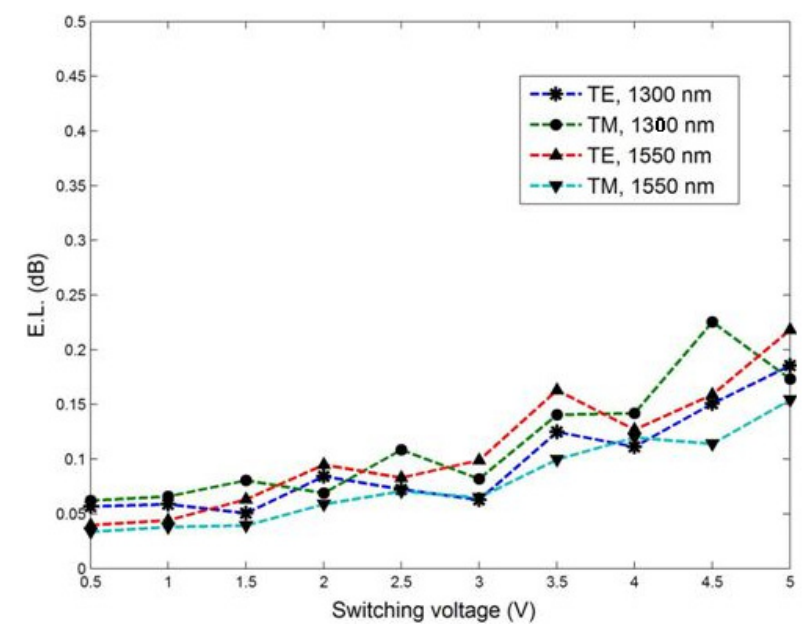

Fig. 3. Variation of excess loss (E.L. in dB) w.r.t applied voltages across electrode regions. 


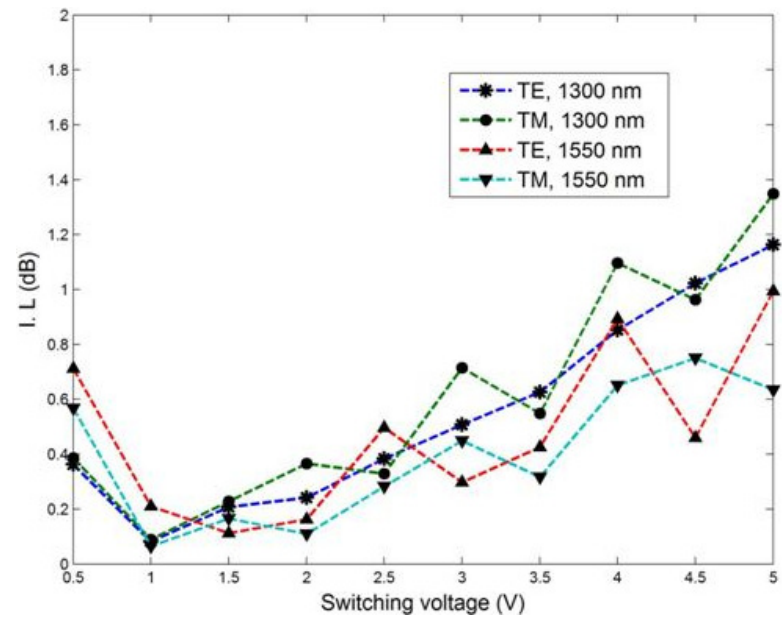

Fig. 4. Variation of insertion loss (I.L. in dB) w.r.t applied voltages across electrode regions.

Figure 5 represents improved trends for the crosstalk levels for both polarizations with lower $(1300 \mathrm{~nm})$ and upper wavelengths $(1550 \mathrm{~nm})$ with an increased index profile, while the branch angle is unchanged $\left(0.1^{\circ}\right)$. Though higher variation in the index shall be only at the cost of a controlled fabrication process and driving voltage requirements i.e. the device fabrication would be complex and costly in such a case. Similarly, trends for enhanced crosstalk levels are noted for the design with a large branch angle as shown in the Fig. 6, while the index profile kept constant at 2.17. Also with increasing the branching angle up to a certain value $\left(0.13^{\circ}\right)$, the CT levels are reduced significantly (even better than $-28 \mathrm{~dB}$, particularly in the case of TE polarized light with a wavelength of $1550 \mathrm{~nm}$ ). The trends of crosstalk levels w.r.t to variation in the index profile, branch angle and switching voltages clearly show the polarization independent operation for a wider range of wavelengths (1300nm-1550nm) in most cases.

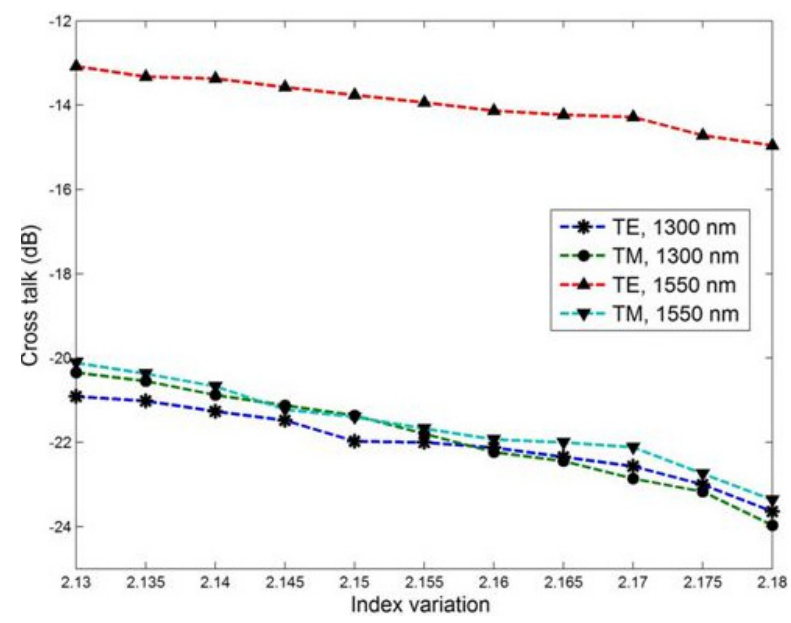

Fig. 5. Variation of crosstalk (in $\mathrm{dB}$ ) w.r.t index variations for TE and TM polarized light signal at the test wavelengths of $1300 \mathrm{~nm}$ and $1500 \mathrm{~nm}$, while the branching angle is fixed at $0.1^{\circ}$.

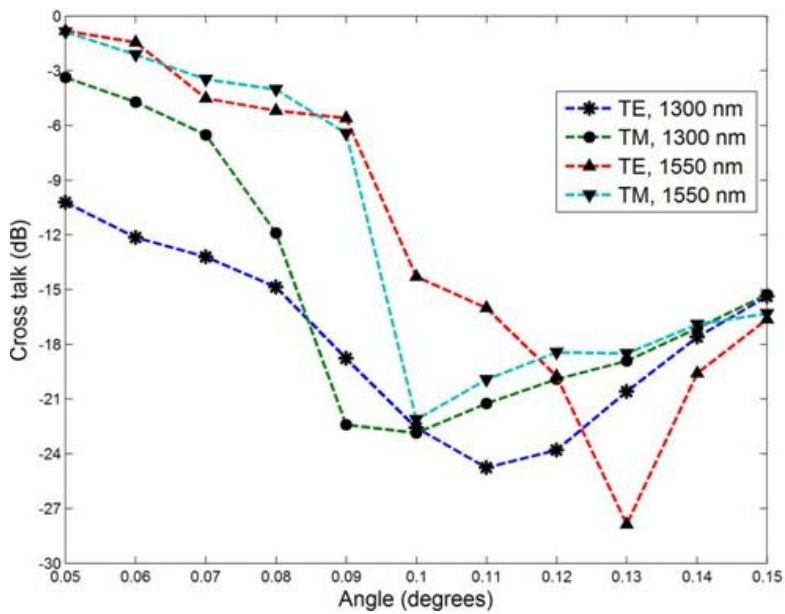

Fig. 6. Variation of crosstalk (in $\mathrm{dB}$ ) w.r.t branch angle for TE and TM polarized light signal at the test wavelengths of $1300 \mathrm{~nm}$ and $1500 \mathrm{~nm}$, while the refractive index is fixed at 2.17 .

In conclusions, we have successfully simulated and analyzed a conventional Digital Optical Switch structure, which complies with Ti in- diffused or proton exchanged Lithium Niobate substrate based Y-shaped wave guiding structure. The results clearly indicate that the device is polarization independent and works for a wide range of wavelengths (1300nm-1550nm). All the calculated switch losses are in the acceptable range and the best crosstalk level is achieved $(-28 \mathrm{~dB}$, in the case of TE polarized light at a wavelength of $1550 \mathrm{~nm}$ ) with minimum possible driving voltage $(1 \mathrm{~V})$ and switch dimensions reported till date with a conventional Y-shaped structure.

Furthermore, crosstalk levels can be suppressed more by adopting slight modifications in the structure such as using s-bend output waveguides, small tapering region or selecting the optimum channel width to streamline the propagating optical power in the desired output arm only.

\section{References}

[1] R. Hauffe, U. Siebel, J. Bruns, K. Peterman, Int. J. Electron. Commun. 55(5), 305 (2001).

[2] M. Iodice, G. Mazzi, L. Sirleto, Opt. Exp. 14(12), 5266 (2006)

[3] L. Sirleto, M. Iodice, F.G. Della Corte, I. Rendina, Opt. Lasers Eng. 45, 458 (2007).

[4] P.J. Duthie, M.J. Wale, Electron. Lett. 27(14), 1265 (1997).

[5] H. Okkayama, M. Kawahara, Electron. Lett. 29(9), 765 (1993).

[6] S.J. Kim, H.S. Jung, H.Y. Lee, Jour. Opt. Soc. Korea 7(4), 253 (2003).

[7] R. Krähenbühl, M.M. Howerton, J. Dubinger, A.S. Greenblatt Jour. Lightwave Tech. 20(1), 92 (2002).

[8] M.N. Kahn, B.I. Miller, E.C. Burrows, C.A. Burrus, IEEE Photon. Technol. Lett. 11, 1250 (1999).

[9] M.K. Pandit, H.P. Chan, K.S. Chiang, S. Ghosh, A.K. Das, Microwave Opt. Techn. Lett. 27(4), 229 (2000).

[10] R. Krähenbühl, W.K. Burns, OSA Photon. Switching 32, 160 (1999).

[11] G. Singh, R.P Yadav, V. Janyani, Jour. Opt. Commun. 31(2), 78 (2010).

[12] Beam Prop 5.1.1, R-Soft Design Group, Inc., Physical Layer Division, 200 Executive Blvd., Ossining, NY 10562. 